\title{
Pregnant women and infants as sentinel populations to monitor prevalence of malaria: results of pilot study in Lake Zone of Tanzania
}

\author{
Ritha A. Willilo ${ }^{1 *}$, Fabrizio Molteni ${ }^{2,3}$, Renata Mandike ${ }^{2}$, Frances E. Mugalura ${ }^{4}$, Anold Mutafungwa',

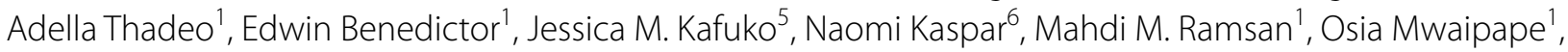 \\ Peter D. McElroy ${ }^{7}$, Julie Gutman ${ }^{8}$, Rajeev Colaco ${ }^{9}$, Richard Reithinger ${ }^{9}$ and Jeremiah M. Ngondi ${ }^{1}$
}

\begin{abstract}
Background: As malaria control interventions are scaled-up, rational approaches are needed for monitoring impact over time. One proposed approach includes monitoring the prevalence of malaria infection among pregnant women and children at the time of routine preventive health facility (HF) visits. This pilot explored the feasibility and utility of tracking the prevalence of malaria infection in pregnant women attending their first antenatal care (ANC) visit and infants presenting at 9-12 months of age for measles vaccination.

Methods: Pregnant women attending first ANC and infants nine to 12 months old presenting for measles vaccination at a non-probability sample of 54 HFs in Tanzania's Lake Zone (Mara, Mwanza and Kagera Regions) were screened for malaria infection using a malaria rapid diagnostic test (RDT) from December 2012 to November 2013, regardless of symptoms. Participants who tested positive were treated for malaria per national guidelines. Data were collected monthly.

Results: Overall 89.9 and $78.1 \%$ of expected monthly reports on malaria infection prevalence were received for pregnant women and infants, respectively. Among 51,467 pregnant women and 35,155 infants attending routine preventive HF visits, 41.2 and $37.3 \%$ were tested with RDT, respectively. Malaria infection prevalence was $12.8 \%$ [95 \% confidence interval (Cl) 11.3-14.3] among pregnant women and $11.0 \%$ (95\% Cl 9.5-12.5) among infants, and varied by month. There was good correlation of the prevalence of malaria among pregnant women and infants at the HF level (Spearman rho $=0.6 ; \mathrm{p}<0.001)$. This approach is estimated to cost $\$ 1.28$ for every person tested, with the RDT accounting for $72 \%$ of the cost.

Conclusions: Malaria infection was common and well correlated among pregnant women and infants attending routine health services. Routine screening of these readily accessible populations may offer a practical strategy for continuously tracking malaria trends, particularly seasonal variation. Positivity rates among afebrile individuals presenting for routine care offer an advantage as they are unaffected by the prevalence of other causes of febrile illness, which could influence positivity rates among febrile patients presenting to outpatient clinics. The data presented here suggest that in addition to contributing to clinical management, ongoing screening of pregnant women could be used for routine surveillance and detection of hotspots.
\end{abstract}

Keywords: Malaria surveillance, Sentinel population, Pregnant women, Infants, Tanzania

*Correspondence: rithawillilo@gmail.com

1 RTI International, Dar es Salaam, Tanzania

Full list of author information is available at the end of the article 


\section{Background}

Populations living in sub-Saharan Africa are at greatest risk for malaria, where approximately $90 \%$ of deaths are estimated to occur, with $70 \%$ of deaths occurring in children under 5 years of age [1]. Tanzania has a substantial burden of malaria. Among children under-five, recent population-based, cross-sectional surveys in 2011/12 documented an overall national prevalence of malaria parasitaemia of $9.2 \%$, ranging from 0 to $31.8 \%$ across the administrative regions [2]. Malaria parasitaemia is similarly prevalent among pregnant women, ranging from 5.5 to $11.5 \%$ [3, 4].

Over the last decade, Tanzania has gradually scaledup malaria control interventions. Insecticide-treated nets (ITNs) have been distributed through various campaigns, including: the Tanzania National Voucher Scheme introduced in 2004 targeting vulnerable groups, such as pregnant women and children aged under five [5]; the children under five catch-up campaign (U5CC) in 2009 [6]; and, the universal coverage campaign (UCC) in 2011 [7]. Artemisinin-based combination therapy (ACT) was introduced as first-line treatment in all public facilities in 2006 and malaria rapid diagnostic tests (RDTs) were introduced in 2009 [8]. Indoor residual spraying (IRS) was introduced in two districts of Kagera Region in 2007 and expanded to 18 districts in the Lake Zone by 2011 [8], where malaria prevalence measured by RDT in children aged 6-59 months was higher (30-41\%) than the rest of mainland Tanzania (18 \%) in 2007-2008 [9]. Following the effective scale-up of multiple malaria control interventions, there has been a $45 \%$ reduction in allcause under-five mortality from 1999 to 2010 [8]. In addition, the Tanzania HIV and Malaria Indicator Survey (THMIS) 2011-12 found that malaria prevalence among children aged 6-59 months had declined to $9 \%$ nationally, and 8, 19 and $26 \%$ in Kagera, Mwanza and Mara Regions, respectively [2]. As malaria control interventions are scaled-up, rational approaches are needed for monitoring impact over time. The 200813 National Malaria Control Programme (NMCP) Monitoring and Evaluation Plan proposed testing of pregnant women and children under 5 years of age attending reproductive and child health $(\mathrm{RCH})$ clinics as a suitable sentinel population for monitoring longitudinal malaria morbidity trends [10]. This study piloted an active screening approach to monitor malaria parasitaemia prevalence in two sentinel populations: (1) pregnant women attending their first ANC visit; and, (2) infants eligible for measles vaccination, at 9-12 months of age attending selected $\mathrm{RCH}$ clinics in Kagera, Mara and Mwanza Regions, as well as to assess the costs of implementing this strategy.

\section{Methods}

\section{Study settings}

The study was conducted from December 2012 to November 2013 in a non-probability sample of 54 health facilities where enhanced surveillance for fast tracking routine malaria data had been implemented and purposively sampled to provide geographical spread across the three regions. The health facilities included 49 (out of131 eligible) health centres (37.4\%) and five (out of 42 eligible) hospitals (11.9\%)) with dedicated RCH clinics in Kagera, Mwanza and Mara Regions in the Lake Zone of Tanzania (Fig. 1). This pilot was initially proposed in the 2008-13 National Malaria Control Program Monitoring and Evaluation Plan to inform future policy [10]. It was considered advantageous because: (1) there is high coverage in antenatal attendances and measles vaccination (over $90 \%$ ); (2) this population represents a homogeneous group than can be followed up longitudinally; (3) this population is easily reachable; (4) these data can provide prospective/longitudinal indications of malaria trends; (5) this system does not require extensive financial resources and is easily implementable under existing routine health care delivery systems; (6) low-levels of training are required; (7) data are easily recorded and reported using a modified information system; (9) there is the potential to add haemoglobin testing in the same facilities to monitor anaemia prevalence; and (10) this data collection system will provide a service to the target population as all positive cases will be treated immediately [10]. Subsequently, the 2014-2020 National Malaria Strategic Plan (MSP) endorsed the policy of screening women at first ANC [11], and in 2015, Tanzania started implementation of routine testing of pregnant women for malaria at first ANC as part of the antenatal profile. In addition, recording and reporting of ANC malaria screening results has been integrated into the routine health management information system (HMIS).

\section{Sample size estimation}

Based on results of a malaria indicator survey done in 2011/12 [2], malaria prevalence in Kagera, Mwanza and Mara Regions was assumed to be approximately 5-15\%. A sample size of 300 people per group (women or infants) per month was chosen to be sufficient to allow estimation of prevalence of malaria of $15 \%$, with a $95 \%$ confidence interval of $11.2-19.6 \%$. As prevalence decreases, if the number of women/infants screened remains constant, the confidence intervals around the estimates would widen.

\section{Malaria parasite detection}

Health facility staff involved in the project were trained using the standard National Malaria Control Programme 


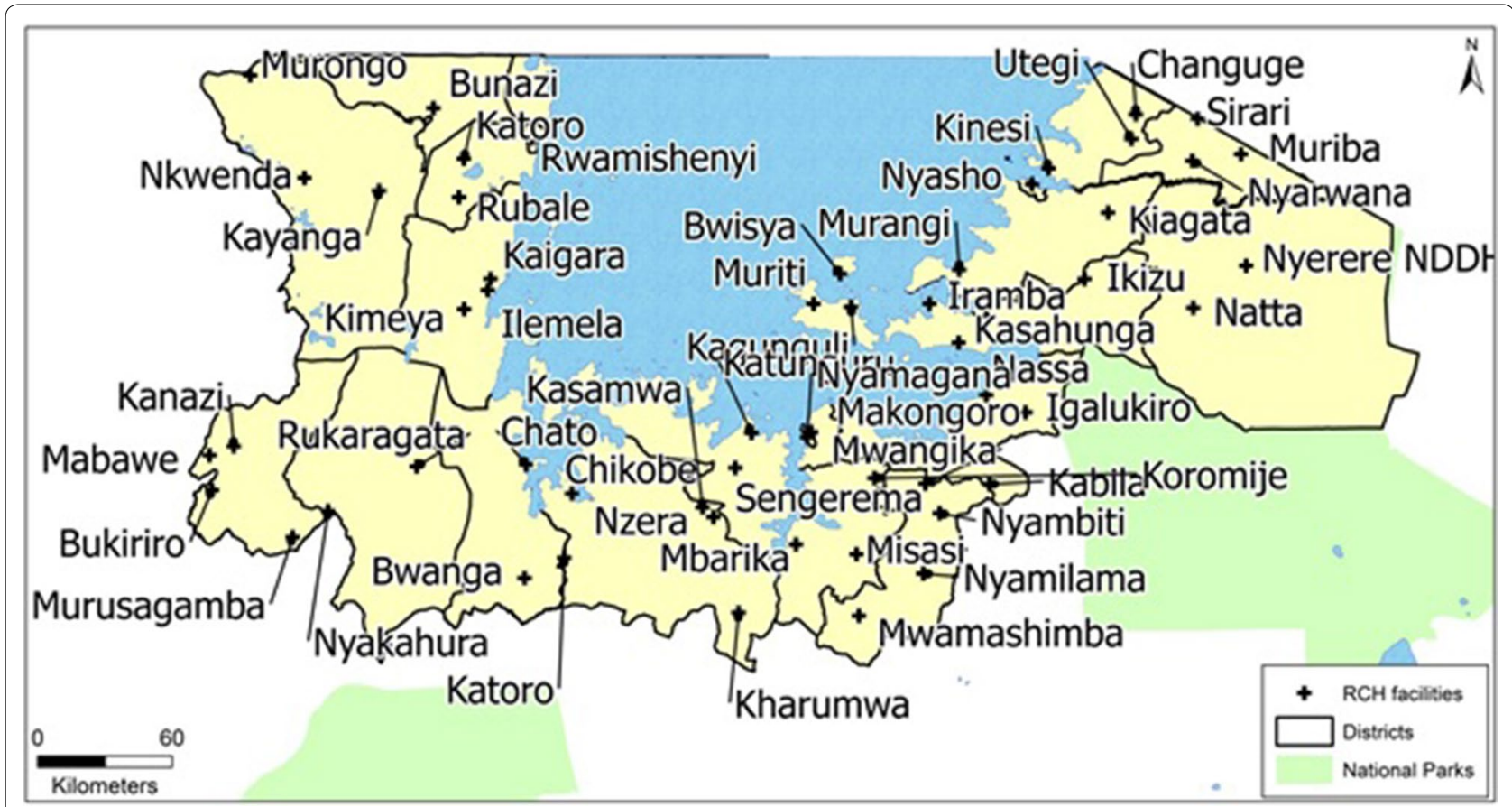

Fig. 1 Geographic location of health facilities participating in the study, Mwanza, Mara and Kagera regions

(NMCP) training manuals [12]. In participating $\mathrm{RCH}$ clinics, pregnant women attending their first antenatal clinic (ANC) visit and infants attending for measles vaccination were recruited to participate in testing for malaria infection with RDT. After obtaining consent, the $\mathrm{RCH}$ nurses obtained capillary blood via finger or heel (for infants) stick for malaria testing using RDT (Malaria Ag P.f/Pan, SD Bioline ${ }^{\circledR}$ ). In addition to completing the routine $\mathrm{RCH}$ clinic register, test results were recorded in a study logbook. No data were collected on symptoms. Participants with positive RDT results were offered immediate treatment with either artemetherlumefantrine, for children and pregnant women in their second or third trimester, or quinine for pregnant women in their first trimester, according to national treatment guidelines.

Data on total number of attendees, total number tested with RDTs and total number of positive RDTs for both pregnant women and infants were aggregated at the facility level on a monthly basis and submitted to the study coordinator for analysis. The district malaria focal person was responsible for collecting and submitting the monthly data summary sheets from his/her respective district.

\section{Analysis of costs}

The direct costs of implementing the $\mathrm{RCH}$ pilot were estimated. The direct cost elements included: training of
$\mathrm{RCH}$ staff; $\mathrm{RCH}$ staff time; consumables (RDT and antimalarials); and, travel expenses for monitoring and supervision. Training of $\mathrm{RCH}$ staff included cost of training venue, accommodation for $\mathrm{RCH}$ staff, printing of study guidelines and reporting forms. Using reported $\mathrm{RCH}$ attendance, it was estimated that on average two eligible infants and three eligible pregnant women attended $\mathrm{RCH}$ clinic daily. Assuming that each test takes approximately $20 \mathrm{~min}$ to perform and read [13], it was estimated that $\mathrm{RCH}$ nurses spent $2 \mathrm{~h}$ per day on RDT tests and recording of test results. The RDT was considered to be the only additional consumable for the testing, and included both purchasing costs ( $\$ 0.55$ average price for Pf/PAN, SD Bioline in 2013) [14] and distribution costs incurred by the Medical Stores Department (MSD) to supply to health facilities. The number of RDTs required was based on the reported $\mathrm{RCH}$ attendance for infants and pregnant women for the 12-month study period, assuming $100 \%$ of attendees were tested. Finally, the travel cost for district staff to travel to health facilities for monitoring, supervision and collection of monthly data summaries was estimated. Costs were converted from Tanzania shilling (TZs) to US dollars (US\$) based on the average exchange rate for 2013 of TZs 1600 per US\$.

\section{Data analysis}

Data were entered and cleaned in a Microsoft Excel spreadsheet. Data analysis was conducted using Stata 
12.0 (Stata Corporation, College Station, TX, USA). Descriptive statistics were used to explore attendance at $\mathrm{RCH}$ clinics and proportion tested for malaria. Multilevel mixed-effects logistic regression models were fitted to adjust confidence intervals of prevalence estimates for clustering of data at health facility levels using the melogit routine in Stata [15]. Differences between proportions were compared using Chi squared tests. Spearman correlation was use to investigate association between proportion of participants tested and malaria positivity and correlation of malaria prevalence estimates between pregnant women and infants. To investigate geographic variation in attendance, malaria testing and malaria prevalence, raster maps were developed using inverse distance weighting (IDW) based on weighted combination of RCH facilities using ArcGIS software (ESRI, Redlands, CA, USA). This technique assumes that malaria data from a certain health facility are from people residing within a certain distance from that facility and therefore the variable being mapped (testing rate and malaria prevalence) decreased in accuracy with increasing distance from its sampled location [16]. Data were examined for spatial autocorrelation using Moran's I test [17, 18].

\section{Results}

\section{Reporting by health facilities}

A total of 54 health facilities ( $31.2 \%$ of eligible sites) were purposively sampled with geographical spread across the three regions. Of the monthly reports expected from the 54 health facilities participating in the pilot, 94.0 and $78.1 \%$ were received for pregnant women and infants, respectively (Table 1 ). The median annual $\mathrm{RCH}$ attendance per health facility was 689 (range 107-4911) and 533 (range 69-1678) for pregnant women and infants, respectively. Mara Region had the most complete monthly reporting for both pregnant women $(95.2 \%)$ and infants (92.9\%), while Mwanza Region had the lowest reporting rates for both populations $(85.6 \%$ for pregnant women and $61.7 \%$ for infants). Figure $2 \mathrm{a}, \mathrm{d}$ show the trends of reporting by month.

\section{$\mathrm{RCH}$ clinic attendance and malaria testing}

A total of 51,467 pregnant women attended their first ANC visit and a total of 35,155 infants attended for measles vaccination at the $54 \mathrm{RCH}$ clinics during this pilot. Overall, 21,184 (41.2 \%) of pregnant women and 13,130 $(37.3 \%)$ of infants were screened for malaria infection (Table 2). Malaria screening rates varied markedly over the study period (Fig. 2b, e) and by location (Fig. 3b, e). Mwanza Region consistently had the lowest testing rates, while in Kagera testing was high in the first 4 months of the study, but declined markedly thereafter (Fig. 2b, e).

\section{Malaria prevalence}

Among those tested, $12.8 \%$ [95 \% confidence interval (CI) 11.3-14.3] of pregnant women and $11.0 \%$ (95\% CI 9.5-12.5) of infants were positive for malaria infection (Table 2). Malaria infection prevalence varied over the study period (Fig. 2c, f) with monthly prevalence of 9.9-14.6 and 5.2-16.7\% among pregnant women and infants, respectively, with marked variation by location (Fig. 3c, e). Malaria infection prevalence was highest in Kagera Region (14.3 and 13.7 \% among pregnant women and infants, respectively) and lowest in Mara Region (11.5 and $7.5 \%$ among pregnant women and infants, respectively).

\section{Variation of health facility attendance, malaria testing and malaria prevalence}

Figure 3 shows the weighted average estimates of health facility attendance, malaria test rates and malaria prevalence among pregnant women and infants based on inverse distance weighing of the health facility observations. There was wide variation across the study areas in both the proportion tested (Fig. 3b, e) and proportion positive (Fig. 3c, f), with evidence of high malaria positivity

Table 1 Summary of reporting by region, December 2012-November 2013

\begin{tabular}{|c|c|c|c|c|}
\hline \multirow[t]{2}{*}{ Characteristic } & \multicolumn{4}{|l|}{ Region } \\
\hline & Kagera & Mara & Mwanza & Total \\
\hline Number of health facilities with RCH clinics & 48 & 49 & 58 & 155 \\
\hline Number of health facilities participating: n (\%) & $18(37.5)$ & $14(28.6)$ & $22(37.9)$ & $54(34.8)$ \\
\hline Number of monthly health facility reports expected & 216 & 168 & 264 & 648 \\
\hline \multicolumn{5}{|l|}{ Pregnant women } \\
\hline Number of reports received & 203 & 160 & 226 & 589 \\
\hline Proportion of reports received (\%) & 94.0 & 95.2 & 85.6 & 90.9 \\
\hline \multicolumn{5}{|l|}{ Infants aged 9-12 months } \\
\hline Number of reports received & 187 & 156 & 163 & 506 \\
\hline Proportion of reports received (\%) & 86.6 & 92.9 & 61.7 & 78.1 \\
\hline
\end{tabular}




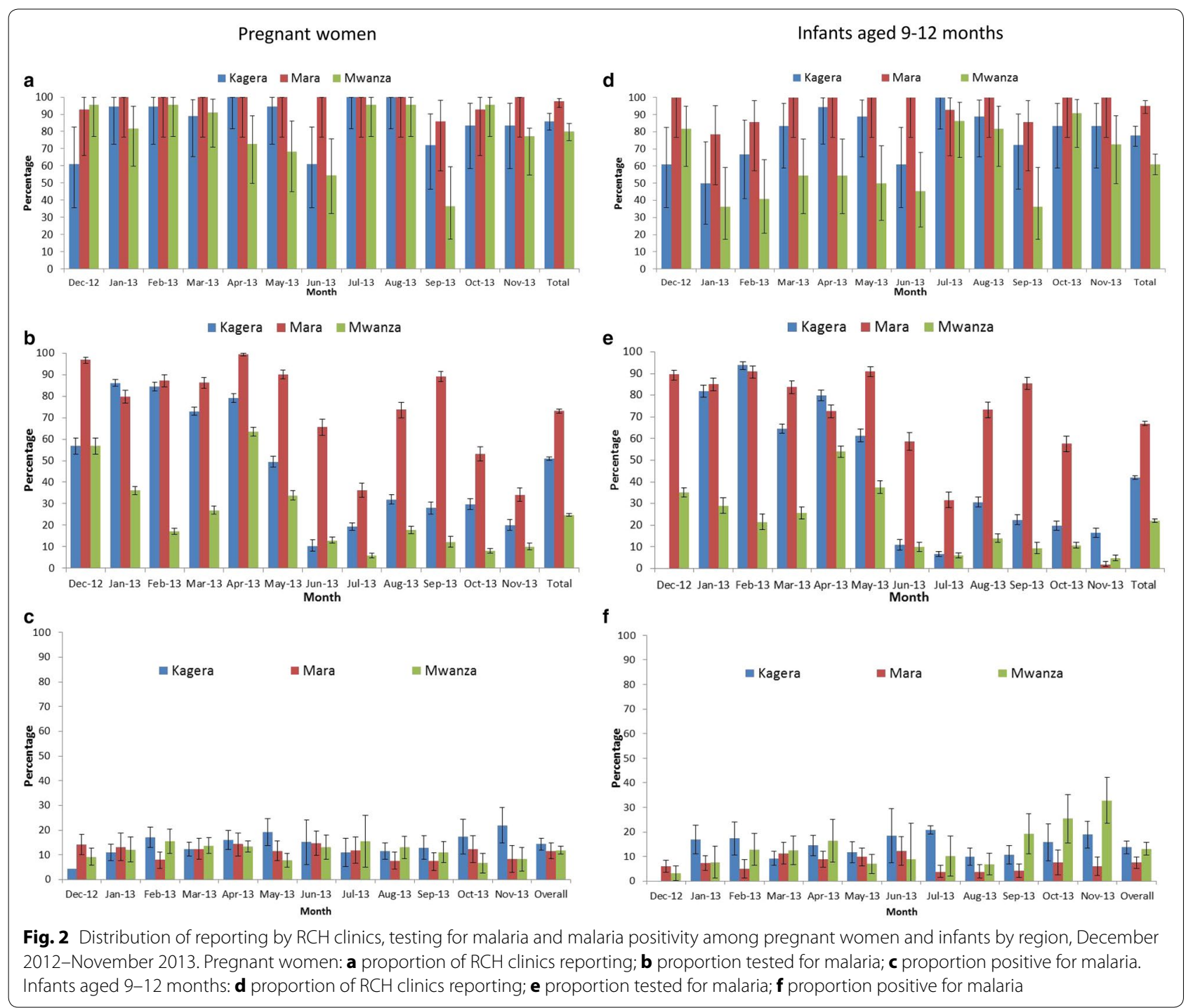

Table 2 Summary of attendance, testing and malaria positivity by region, December 2012-November 2013

\begin{tabular}{|c|c|c|c|c|c|c|c|c|}
\hline \multirow[t]{2}{*}{ Characteristic } & \multicolumn{4}{|c|}{ Pregnant women } & \multicolumn{4}{|c|}{ Infants aged 9-12 months } \\
\hline & Kagera & Mara & Mwanza & Total & Kagera & Mara & Mwanza & Total \\
\hline $\begin{array}{l}\text { Number of partici- } \\
\text { pants attending } \\
\text { clinic }\end{array}$ & 16,844 & 9177 & 25,446 & 51,467 & 13,798 & 8050 & 13,307 & 35,155 \\
\hline Number tested & 8283 & 6701 & 6200 & 21,184 & 4823 & 5619 & 2688 & 13,130 \\
\hline $\begin{array}{l}\text { Proportion of } \\
\text { participants } \\
\text { tested (\%) }\end{array}$ & 49.2 & 73.0 & 24.4 & 41.2 & 35.0 & 69.8 & 20.2 & 37.3 \\
\hline Number positive & 1158 & 782 & 733 & 2673 & 668 & 417 & 360 & 1445 \\
\hline $\begin{array}{l}\text { Malaria positiv- } \\
\text { ity: } \%(95 \% \mathrm{Cl})\end{array}$ & $14.3(11.9,16.8)$ & $11.5(8.4,14.7)$ & $11.9(10.2,13.6)$ & $12.8(11.3,14.3)$ & $13.7(11.1,16.3)$ & $7.5(5.2,9.8)$ & $13.1(10.5,15.8)$ & $11.0(9.5,12.5)$ \\
\hline
\end{tabular}


(hotspots) in defined geographic locations. There was good correlation between prevalence of malaria among pregnant women and infants across the geographic distribution of reporting sites, especially in the high transmission areas (Fig. 3c, f). Moran's I test showed that there was no spatial autocorrelation of health facility level malaria prevalence among pregnant women $(\mathrm{p}=0.155)$ and infants $(\mathrm{p}=0.353)$. The health facility prevalence of malaria in pregnant women was correlated with malaria prevalence in infants (Spearman rho $=0.6 ; \mathrm{p}<0.001$ ). There was no correlation between malaria prevalence and proportion of participants tested for malaria among pregnant women (Spearman rho $=-0.04 ; \mathrm{p}=0.8$ ) or infants (Spearman rho $=-0.2 ; \mathrm{p}=0.3$ ).

\section{Comparison of prevalence with malaria positivity in outpatient department}

The malaria positivity rate in the outpatient department (OPD) of the participating facilities was collected in order to compare this to the prevalence among those attending $\mathrm{RCH}$ services. Overall, malaria positivity rates in the OPD varied by month and region, ranging from 15.6 to $53.0 \%$, with an average of 42.4, 34.8 and $27.4 \%$ in Kagera, Mara and Mwanza regions, respectively (Fig. 4). Overall, there was a positive correlation between OPD positivity rate and positivity for both pregnant women and infants, although the correlation was only modest (Spearman's rho $=0.3, p<0.001$ for pregnant women and Spearman's rho $=0.4, \mathrm{p}<0.001$ for infants) (Fig. $5 \mathrm{a}, \mathrm{b})$.

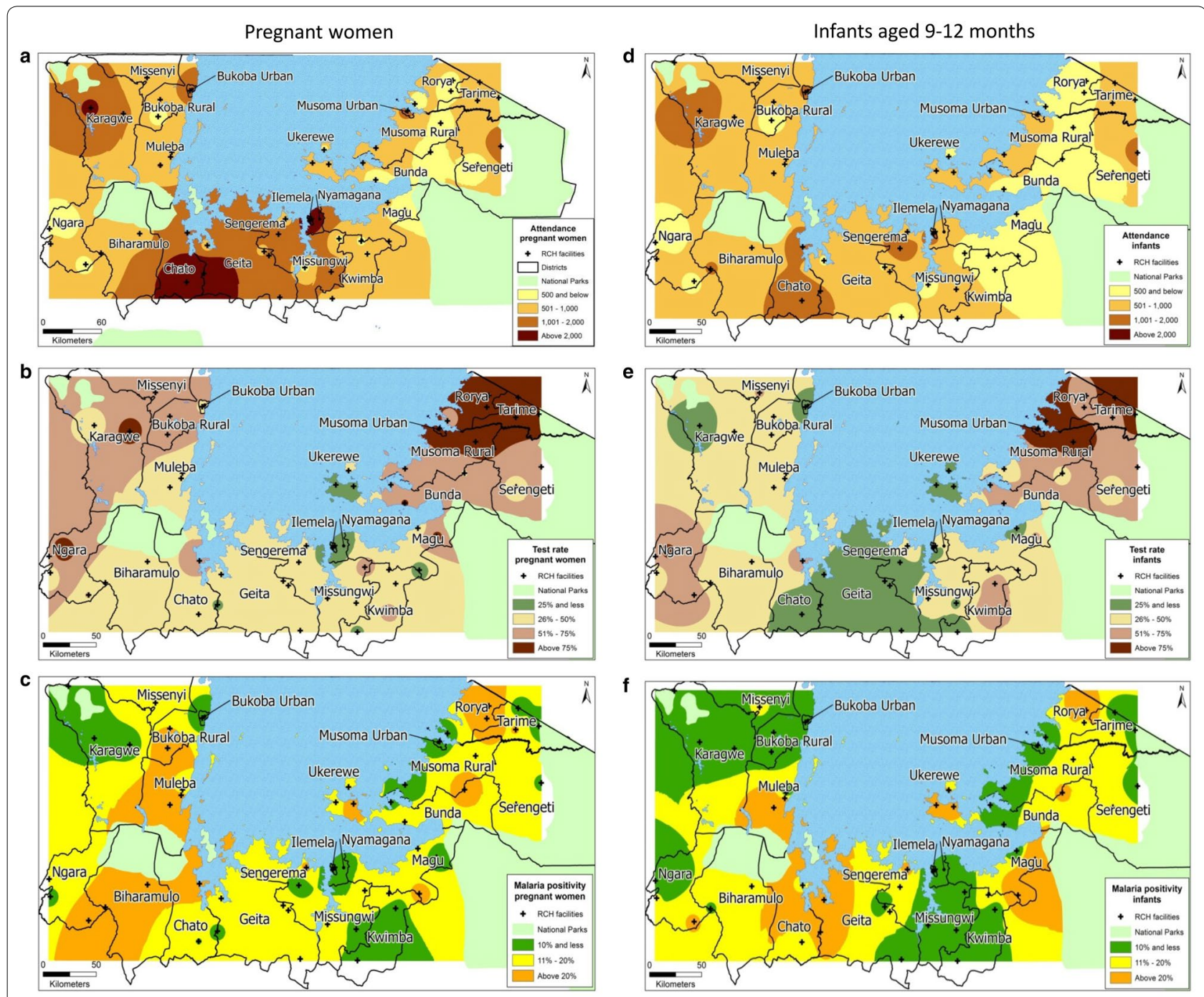

Fig. 3 Geographical variation in RCH clinics attendance, malaria testing and malaria prevalence among pregnant women and infants by district. Pregnant women: a number attending RCH clinics; b proportion tested for malaria; $\mathbf{c}$ proportion positive for malaria. Infants aged 9-12 months: $\mathbf{d}$ number attending RCH clinics; e proportion tested for malaria; $\mathbf{f}$ proportion positive for malaria 


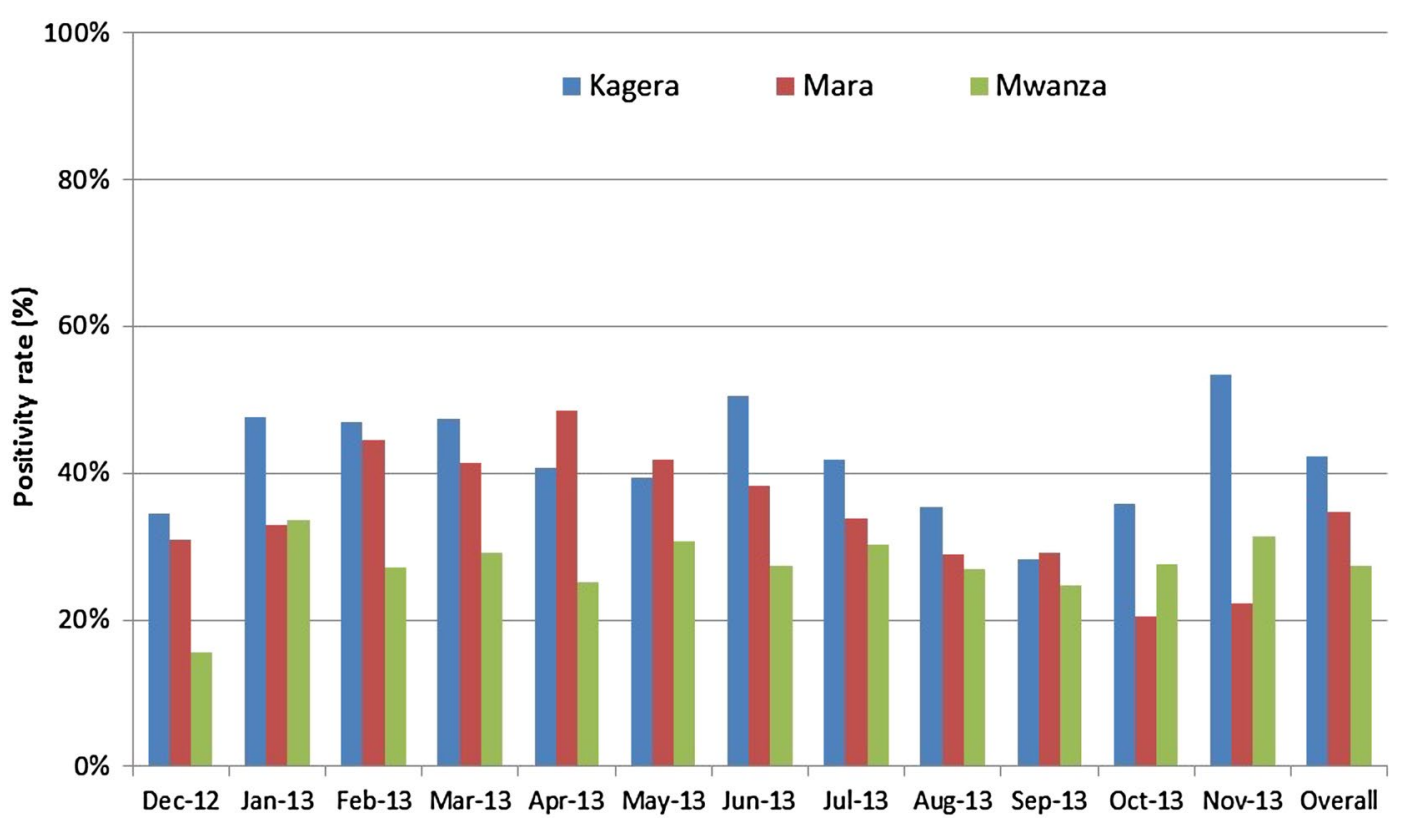

Fig. 4 Malaria positivity among patients attending outpatient clinic in 54 health facilities participating in sentinel surveillance

\section{Costs analysis}

The estimated direct costs of undertaking this study, based on RCH attendance, are summarized in Table 3. The average total cost of testing pregnant women and infants per health facility was estimated at US\$2060.59 per year. The key cost driver was the cost of purchase and delivery of RDTs to the health facilities that accounted for $72 \%$ of the total direct costs. It was estimated that this approach would cost US $\$ 1.28$ for every person tested and the cost per positive test was estimated at US\$10.70.

\section{Discussion}

In an area of Tanzania with high malaria transmission where intensive vector control interventions (IRS and ITNs) were scaled up and complemented by universal access to ACT and RDT, nearly one in eight pregnant women and one in ten infants attending $\mathrm{RCH}$ clinics

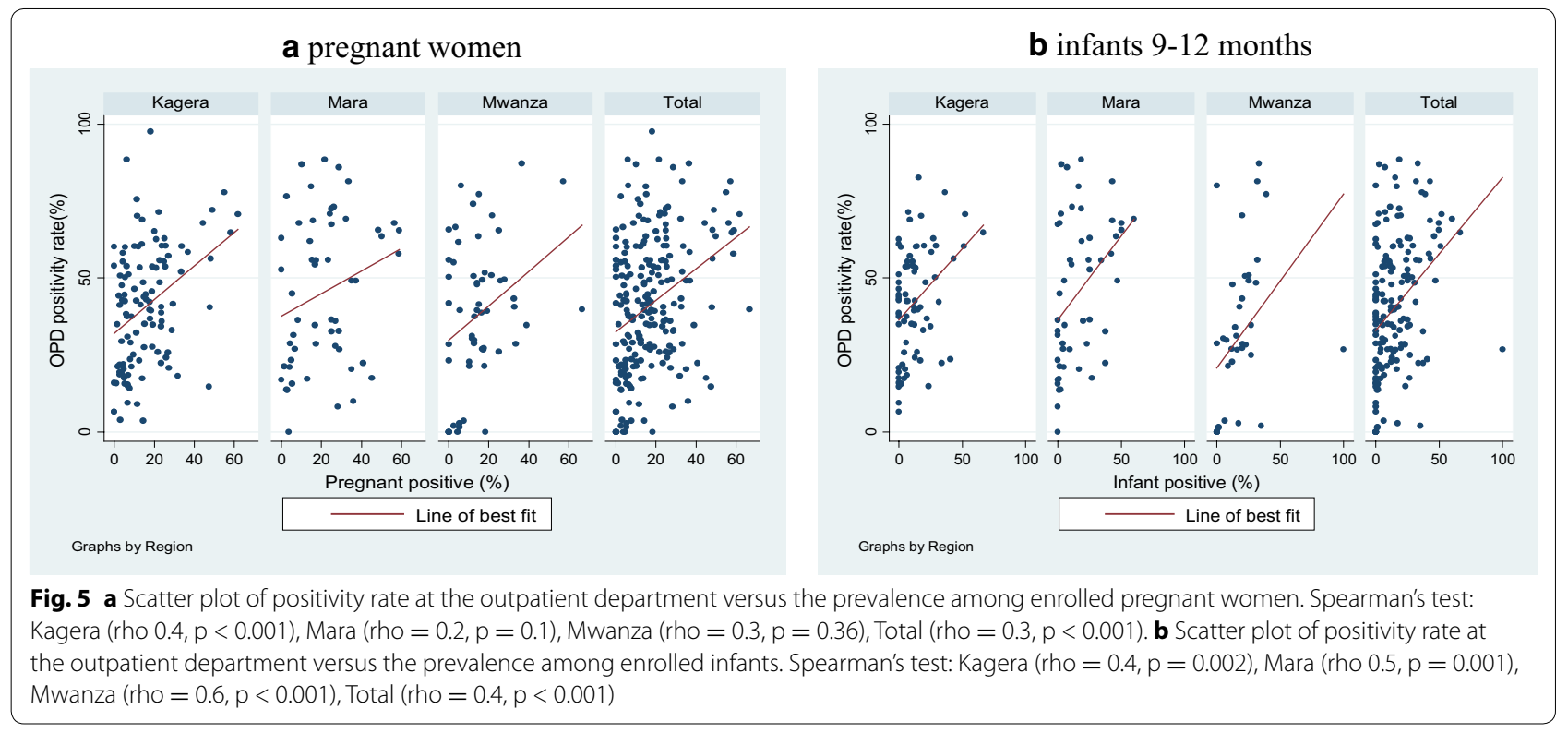


Table 3 Direct costs associated with the testing of pregnant women and children in RCH clinics

\begin{tabular}{lccc}
\hline Cost elements & Annual cost (US\$) & Proportion of total cost (\%) \\
\cline { 2 - 4 } & \multicolumn{1}{l}{ Total for $\mathbf{5 4}$ health facilities } & Average per health facility & \\
\hline Training of RCH staff & 7656.25 & 141.78 & 7 \\
Personnel: RCH nurses & 3240.00 & 60.00 & 3 \\
Consumables (RDT) & $80,125.35$ & 1483.80 & 72 \\
Travel for monitoring and supervision & $20,250.00$ & 375.00 & 18 \\
Total & $111,271.60$ & 2060.59 & 100 \\
\hline
\end{tabular}

$R C H$ reproductive and child health; $R D T$ rapid diagnostic test

a Consumables based on reported attendance (assumes $100 \%$ test rate for both pregnant women and infants). Cost of RDT based on average price for 2013 [14]

were positive for malaria infection. In general, the trends over time and space were similar for the two populations. Mapping the prevalence by location revealed the presence of localized high transmission hotspots for both the pregnant women and infants. While malaria test positivity rates from the OPD provide a source of data, which could be used to identify malaria hotspots, these data are influenced by other causes of fever. This study piloted a new approach for assessing populationbased prevalence of malaria through targeted screening of readily accessible, asymptomatic populations (pregnant women and infants) that have high attendance at $\mathrm{RCH}$ clinics (95.9\% of pregnant women attend at least one ANC visit and $74.6 \%$ of infants attend for measles vaccination) [19]. Compared to test positivity rate data obtained from the OPD, testing of a sentinel population (including both symptomatic and asymptomatic individuals) provides a more stable estimate over time, which is unaffected by other circulating illnesses. This data thus provides a better measure for monitoring disease trends over time. Cross-sectional household surveys are typically used to provide this data, however, these are costly and are performed at widely spaced intervals, which does not allow for fine scale tuning of intervention delivery in the interim. Ongoing, routine screening of readily accessible populations may offer a practical strategy for continuous monitoring to identify malaria hotspots and track the progress of malaria control over time. These data complement the data from the OPD, thus combining these sources could provide a more comprehensive understanding of the full epidemiologic picture.

In Tanzania, sentinel population testing of pregnant women and children under 5 years of age attending $\mathrm{RCH}$ clinics was initially proposed in the 2008-13 Malaria Monitoring and Evaluation Plan [10]. Following the pilot study, the 2014-2020 National Malaria Strategic Plan set a strategic intervention to establish countrywide longitudinal vigilance of malaria parasitaemia in sentinel populations: pregnant women and infants at $\mathrm{RCH}$ clinics, and school-age children [11]. While data obtained from these sentinel populations would not obviate the need for periodic cross-sectional population-based surveys to measure coverage of interventions, the available data suggest that the prevalence of anaemia and parasitaemia among children presenting to the health facility for routine immunization is correlated with that among children aged 6-30 months detected by household surveys [11]. Similarly, data from a recent meta-analysis by van Eijk found that the prevalence of malaria among pregnant women was strongly correlated with that from household surveys of children [20], suggesting that either of these populations could be used to provide ongoing information about community level prevalence. From a biological standpoint, monitoring of infants may be preferable to monitoring adult women. The prevalence among infants nine to 12 months of age should closely mirror that of all children 6-59 months. Since infants have not yet developed significant immunity, these infections are likely to have been recently acquired. Furthermore, infants are less likely to have travelled than pregnant women. Thus, the prevalence of infection among infants may be more likely to represent recent and local transmission dynamics than that in adult women. However, pregnant women are more likely to be infected with malaria than nonpregnant women, and are at greater risk for severe disease than non-pregnant women [21]. This is particularly true for primigravid women, who are at increased risk of severe disease, with higher risk of severe anaemia and maternal death, higher rates of miscarriage, intrauterine demise, premature delivery, low-birth-weight neonates, and neonatal death compared to multigravid women [22]. Identifying and treating infected pregnant women with asymptomatic infections who may otherwise have gone untreated may have the added benefit of improving birth outcomes [23]. In addition, this may have a benefit on transmission, as it has been suggested that pregnant women may be a reservoir of transmission [24]. Finally, during the first ante-natal care visit, a panel of blood 
tests is already performed, whereas children presenting for measles immunization would not routinely have a blood draw. For these reasons, in Tanzania, testing of pregnant women was felt to be a better choice for routine monitoring than infants, and in 2015, Tanzania started implementation of routine testing of pregnant women for malaria at first ANC as part of the antenatal profile [25]. In addition, recording and reporting of ANC malaria screening results has been integrated into the routine health management information system (HMIS).

The study estimated the direct costs associated with the sentinel population screening pilot. The costs included training, staff time required to perform and record the test, test kits, and travel expenses for monitoring and supervision. These estimates suggest that nearly threequarters of direct costs were attributable to purchase and delivery costs for RDTs. Nonetheless, reporting of ANC malaria testing through routine HMIS, and integration of RDT quality assurance monitoring and supervision with other routine supervisory activities, that are undertaken on a quarterly basis by the district teams, would reduce these costs.

This study has a number of potential limitations. The low rate of testing in Kagera and Mwanza is a potential source of bias and may have underestimated the results. The low testing rates were largely attributed to stock-outs of RDTs during the study period. Several reasons were reported by RCH clinics to explain frequent RDT stockouts, including: weak quantification and forecasting by health facilities, delayed delivery of RDTs to health facilities by MSD, and stock out at MSD central stores. The analysis suggests that malaria prevalence was not correlated with the proportion of participants tested; thus the low testing rate is not expected to have biased the results. Lack of reporting was partly related to testing activity because $\mathrm{RCH}$ clinics did not submit any reports where testing was absent due to RDT stock-outs. It is likely that the low reporting rates, especially among infants in Mwanza Region, may have biased the results. In this study, participating health facilities were purposively selected to provide a geographically spread sample, but were not probabilistically selected to support parametric statistics and generalizable estimates. While this may be a potential source of bias, sampling is not likely to have influenced reporting rates and RDT stock-outs. Finally, history of recent symptoms for the persons tested was not recorded, as it was not part of the routine ANC register, making it impossible to know if those who tested positive had fever or other malaria symptoms in recent days (but were asymptomatic at time of the $\mathrm{RCH}$ visit). Finally, there is evidence to suggest that the HRP-2/pLDH RDTs are not sufficiently sensitive to diagnose very low density malaria infections in asymptomatic pregnant women [26], thus some infections may be missed. Nonetheless, if this screening activity also worked to detect very early cases of uncomplicated malaria, then this approach could benefit low transmission areas where additional efforts are needed to reinforce passive case detection.

\section{Conclusions}

Routine screening of pregnant women at first ANC and infants presenting for measles vaccination may offer a practical approach for ongoing monitoring to track the progress of malaria control over time. This strategy provides benefit to the tested individual by identifying and treating those with asymptomatic but detectable parasitaemia. In addition, the continuous data generated down to the sub-district level allows for targeting malaria control interventions according to the level of transmission. Of the two groups, testing of pregnant women is likely to be less disruptive to clinic flow, as pregnant women already have blood drawn for routine ANC screening, but infants may provide a more precise estimate of transmission variability across sites and over time. Malaria screening of pregnant women at first ANC should be considered as a practical method for continuous routine surveillance of malaria prevalence at the sub-district level.

\section{Abbreviations}

ANC: antenatal clinic; ACT: artemisinin combination therapy; HMIS: health management information system; IDW: inverse distance weighting; IRS: indoor residual spraying; ITN: insecticide-treated net; LLIN: long-lasting insecticidal nets; RDT: rapid diagnostic test; MSD: medical stores department; NMCP: National Malaria Control Programme; OPD: outpatient department; $\mathrm{RCH}$ : reproductive and child health; THMIS: Tanzania HIV and Malaria Indicator Survey; WHO: World Health Organization.

\section{Authors' contributions}

RAW, RM, FM, FEM, JMK, NK, MMR, PM and JG designed the study. FEM, AM, AT and $E B$ undertook data collection. RAW, OM, RC, RR and JMN undertook data analysis. RAW, RC, RR and JMN drafted the manuscript which all authors edited and approved. All authors read and approved the final manuscript.

\section{Author details \\ ${ }^{1}$ RTI International, Dar es Salaam, Tanzania. ${ }^{2}$ National Malaria Control Pro- gramme, Dar es Salaam, Tanzania. ${ }^{3}$ Swiss Tropical and Public Health Institute, Dar es Salaam, Tanzania. ${ }^{4}$ Sengerema Health Institute, Sengerema, Tanzania. ${ }^{5}$ President's Malaria Initiative/United States Agency for International Develop- ment, Abuja, Nigeria. ${ }^{6}$ President's Malaria Initiative/United States Agency for International Development, Dar es Salaam, Tanzania. ${ }^{7}$ President's Malaria Initiative and Malaria Branch, Centers for Disease Control and Prevention, Atlanta, USA. ${ }^{8}$ Malaria Branch, Division of Parasitic Diseases and Malaria, Cent- ers for Disease Control and Prevention, Atlanta, GA, USA. ${ }^{9}$ RTI international, Washington DC, USA.}

\section{Acknowledgements}

We are grateful to the staff and reproductive and child health clinics who implemented the study and district malaria focal persons (DMFP) in Lake Zone for supervising the study.

Competing interests

The authors declare that they have no competing interests. 


\section{Availability of data and material}

The datasets generated during the current study are available from the corresponding author on reasonable request.

\section{Disclaimer}

The findings and conclusions presented in this manuscript are those of the authors and do not necessarily reflect the official position of the US President's Malaria Initiative, US Agency for International Development or US Centers for Disease Control and Prevention.

\section{Ethical approval and consent to participate}

The study protocol was approved by the institutional review boards of US Centers for Disease Control and Prevention (CDC) and Tanzanian Medical Research Coordinating Committee. Consent for study participation was obtained from pregnant women and from the adults (mother, caregiver or guardian) who brought infants for measles vaccination. Infants brought for measles vaccinations by persons below the age of 18 years were excluded from the study. No individual patient information was reported.

\section{Funding}

This study was made possible through support provided to the Tanzania Vector Control Scale-up Project (Cooperative Agreement 621-A-00-10-00015-00) by the President's Malaria Initiative (PMI) via the US Agency for International Development (USAID) and an inter-agency agreement with the US Centers for Disease Control and Prevention (CDC).

Received: 27 January 2016 Accepted: 18 July 2016

Published online: 29 July 2016

\section{References}

1. WHO. Malaria fact sheet. Geneva: World Health Organization; 2015. http:// www.who.int/mediacentre/factsheets/fs094/en/. Accessed 15 Apr 2016.

2. Tanzania Commission for AIDS (TACAIDS), Zanzibar AIDS Commission (ZAC), National Bureau of, Statistics (NBS), Office of the Chief Government Statistician (OCGS), and ICF International. Tanzania HIV/AIDS and Malaria Indicator Survey 2011-12 [Internet]. Dar es Salaam, Tanzania: TACAIDS, ZAC, NBS, OCGS, and ICF International; 2013. https://dhsprogram.com/ pubs/pdf/AIS11/AIS11.pdf. Accessed 25 July 2016.

3. Zinga M. Malaria parasitaemia and associated preventive factors among pregnant women in Nyamagana and Misungwi districts, Mwanza region, 2012. Dar es Salaam: Muhimbili University of Health and Allied Sciences; 2012

4. Mosha TC, Ntarukimana D, John M. Prevalence of congenital malaria among neonates at Morogoro Regional Hospital, Morogoro, Tanzania. Tanzan J Health Res. 2010;12:241-8.

5. Mushi AK, Schellenberg JR, Mponda H, Lengeler C. Targeted subsidy for malaria control with treated nets using a discount voucher system in Tanzania. Health Policy Plan. 2003;18:163-71.

6. Bonner K, Mwita A, McElroy PD, Omari S, Mzava A, Lengeler C, et al. Design, implementation and evaluation of a national campaign to distribute nine million free LLINs to children under five years of age in Tanzania. Malar J. 2011;10:73.

7. Renggli S, Mandike R, Kramer K, Patrick F, Brown NJ, McElroy PD, et al. Design, implementation and evaluation of a national campaign to deliver 18 million free long-lasting insecticidal nets to uncovered sleeping spaces in Tanzania. Malar J. 2013;12:85.

8. Global Partnership to Roll Back Malaria. Focus on mainland Tanzania. Progress and impact series_country reports; $\mathrm{n} 3$. Geneva: World Health Organization; 2011

9. Tanzania Commission for AIDS (TACAIDS), Zanzibar AIDS Commission (ZAC), National Bureau of Statistics (NBS), Office of the Chief Government Statistician (OCGS), ICF International. Tanzania HIV/AIDS and malaria indicator survey 2008-2007. Dar es Salaam: Tanzania Commission for AIDS (TACAIDS); 2008.
10. Ministry of Health and Social Welfare. National Malaria Control Programme Monitoring and Evaluation Plan 2008-2013. http://www. natnets.org/attachments/article/65/Monitoring\%20\&\%20Evaluation\%20 Plan\%202008-2013.pdf. Accessed 14 Jan 2016.

11. National Malaria Control Programme. National malaria strategic plan 2014-2020. Dar es Salaam: National Malaria Control Programme; 2014.

12. National Malaria Control Programme. Learners' manual for malaria rapid diagnostic test: training on malaria rapid diagnostic test to health care workers. 1st ed. Dar es Salaam: Ministry of Health and Social Welfare; 2010.

13. Rapid diagnostic tests: how they work. http://www.cdc.gov/malaria/ malaria_worldwide/reduction/dx_rdt.html. Accessed 19 May 2016.

14. USAID. Deliver project. Task Order 7. http://deliver.jsi.com/dlvr_content/ resources/allpubs/toandiqc/TO7SemiAnnuFY2014.pdf. Accessed 29 Jan 2015.

15. Multilevel mixed-effects logistic regression. http://www.stata.com/manuals13/memelogit.pdf. Accessed 12 Apr 2015.

16. How inverse distance weighted interpolation works. http://pro.arcgis, com/en/pro-app/help/analysis/geostatistical-analyst/how-inversedistance-weighted-interpolation-works.htm. Accessed 27 Jan 2015.

17. Moran P. The interpretation of statistical maps. J R Stat Soc B. 1948;10:243-51.

18. Exploratory spatial data analysis using Stata. http://www.stata.com/meeting/germany12/abstracts/desug12_pisati.pdf. Accessed 9 Apr 2015.

19. National Bureau of Statistics (NBS) and ICF Macro. Tanzania demographic and health survey 2010. Dar es Salaam: NBS and ICF Macro; 2010. p. 2011.

20. Mathanga DP, Campbell CH Jr, Vanden Eng J, Wolkon A, Bronzan RN, Malenga GJ, et al. Comparison of anaemia and parasitaemia as indicators of malaria control in household and EPI-health facility surveys in Malawi. Malar J. 2010;9:107.

21. Desai M, ter Kuile FO, Nosten F, McGready R, Asamoa K, Brabin B, et al. Epidemiology and burden of malaria in pregnancy. Lancet Infect Dis. 2007:7:93-104

22. Schantz-Dunn J, Nour NM. Malaria and pregnancy: a global health perspective. Rev Obstet Gynecol. 2009;2:186-92.

23. De Beaudrap P, Turyakira E, White LJ, Nabasumba C, Tumwebaze B, Muehlenbachs $A$, et al. Impact of malaria during pregnancy on pregnancy outcomes in a Ugandan prospective cohort with intensive malaria screening and prompt treatment. Malar J. 2013;12:139.

24. Boudova S, Cohee LM, Kalilani-Phiri L, Thesing PC, Kamiza S, Muehlenbachs $\mathrm{A}$, et al. Pregnant women are a reservoir of malaria transmission in Blantyre, Malawi. Malar J. 2014;13:506

25. National Malaria Control Programme. Learners' manual for malaria rapid diagnostic test: training on malaria rapid diagnostic test to health care workers. 2nd ed. Dar es Salaam: Ministry of Health and Social Welfare; 2014.

26. Umbers AJ, Unger HW, Rosanas-Urgell A, Wangnapi RA, Kattenberg $J \mathrm{H}$, Jally S, et al. Accuracy of an HRP-2/panLDH rapid diagnostic test to detect peripheral and placental Plasmodium falciparum infection in Papua New Guinean women with anaemia or suspected malaria. Malar J. 2015;14:412.

\section{Submit your next manuscript to BioMed Central and we will help you at every step:}

- We accept pre-submission inquiries

- Our selector tool helps you to find the most relevant journal

- We provide round the clock customer support

- Convenient online submission

- Thorough peer review

- Inclusion in PubMed and all major indexing services

- Maximum visibility for your research

Submit your manuscript at www.biomedcentral.com/submit 